\title{
Obesity, depression, and alcohol linkages among women
}

\section{Introduction}

The terms "overweight" and "obesity" that refer to a body weight that is greater than what is considered to be healthy, ${ }^{1}$ commonly have a highly negative health connotation. A condition that appears largely preventable, rather than only genetically determined,-obesity remains one of the world's most serious seemingly intractable health conditions. Currently considered a global epidemic because the worldwide obesity population is not only expanding in numbers, but also in weight, ${ }^{2-6}$ many attempts have been made to examine the causes of adult obesity, known to shorten life, as well as life quality. This brief examines data concerning the possible link between alcohol intake and obesity in women. The possible link to depression is also examined. The rationale for focusing on women is the 2014 report by Charo et al., ${ }^{7}$ that obesity rates remain high and fundamentally unchanged among women and girls in the United States and that this situation seriously impacts health outcomes across the reproductive lifespan. Alcohol usage was the topic of interest, given the possible relevant relationship between food addictions and obesity, depression and alcohol use, and depression and obesity, among women, which has not been well studied.

\section{Method}

Data were sought on PUBMED and the internet using the key terms, obesity, alcohol, women, and depression. English language reports dating back to 1980 were sought, regardless of type. Selected were those pertinent to the ensuing discussion, and these are presented in narrative form, given the lack of depth on any of the specific topics of interest.

\section{Results}

\section{General observations}

Despite the paucity of data pertaining to the current topics of interest, some of these research efforts do show women tend to be more vulnerable than men in terms of becoming or being obese, especially with regards to extreme obesity, ${ }^{6}$ although reasons for this are not clear. However, because women also show greater levels of disability from complications related to obesity, some studies have specifically focused on examining gender-related factors that may explain women's higher rates of obesity. In this regard some data point to the presence of depression or depressive symptoms, given that for every age group, women with depression appear more likely to be obese than women without depression. The proportion of adults with obesity also rose as the severity of depressive symptoms increased in a related analysis. ${ }^{8}$ Other data showed $55 \%$ of adults taking antidepressant medications, still reported moderate to severe depressive symptoms, and were obese, suggesting other obesity associated factors should be explored. One that has also been explored is alcohol.

\section{Specific observations}

Although the link between alcohol intake and obesity is not conclusive by any means, and different conclusions prevail, this body of research shows several important findings-
Volume 8 Issue 5 - 2018

\author{
Ray Marks \\ Department of Health and Behavior Studies, Program in Health \\ Education, Columbia University, Teachers College, and School \\ of Health and Professional Studies, Department of Health, \\ Physical Education \& Gerontological Studies and Services, City \\ University of New York, USA
}

\begin{abstract}
Correspondence: Ray Marks, Department of Health and Behavior Studies, Program in Health Education, Columbia University, Teachers College, and School of Health and Professional Studies, Department of Health, Physical Education \& Gerontological Studies and Services, City University of New York, York College, New York, USA, Tel + I (212) 678-3445, Fax +I(2I2)678-8259, Email rm226@columbia.edu
\end{abstract}

Received: August 08, 2018 | Published: September 05, 2018

a. Globally, alcohol misuse is the fifth leading risk factor for premature death and disability; among people between the ages of 15 and 49

b. Recreational alcohol intake is a widespread activity globally and alcohol energy $(7 \mathrm{kcal} / \mathrm{g})$ can be a contributing factor to weight gain if not compensated for. ${ }^{9}$

c. The only predictor of obesity in Serbian women ages 20 or above was alcohol use. ${ }^{10}$

d. Other recent research further shows that in 2001-2002, women with a family history of alcoholism had a $49 \%$ higher odds of being obese than those without a family history (odds ratio, 1.48; $95 \%$ confidence interval, 1.36-1.61; $\mathrm{P}<.001$ ), a highly significant increase $(\mathrm{P}<.001)$, from the odds ratio of $1.06(95 \%$ confidence interval, 0.97-1.16) estimated for 1991-1992. For men, however, the association was significant (odds ratio, 1.26; 95\% confidence interval, 1.14-1.38; $\mathrm{P}<.001$ ) but not as strong. The association and secular trend for women was also robust after adjustment for covariates, including sociodemographic variables, smoking status, alcohol use, alcohol /drug dependence, and major depression, but not for men. ${ }^{6}$ Additional related data reveal adults with depression are more likely to be obese than adults without depression according to the CDC/NCHS, National Health and Nutrition Examination Survey, 2005-2010 results, ${ }^{8}$ possibly because the anti depressant medication is a risk factor for obesity, among other factors. These include emotional factors, genetic factors, addictive tendencies, social and environmental factors, among others.

Unsurprisingly, evidence linking depression, obesity, and alcoholism also prevails. These data taken from 776 adults show -:

a) Women with alcohol disorders at age 24 were $4 x$ more likely to be obese at 27 .

b) Women who were obese at 27 were twice as likely to be depressed by age 30 .

c) Women who depressed at age 27 were at increased risk for alcohol disorders at $30 .{ }^{11}$ 
In their study, McCartney et al., ${ }^{11}$ also found that among women, depression was positively associated with later alcohol use disorders (ages 27 to 30: $\mathrm{OR}=3.11$ ). As well, they found alcohol use disorders prospectively predicted obesity (ages 24 to 27 : $\mathrm{OR}=3.84$ ). Obesity predicted depression from ages 27 to 30 among women $(\mathrm{OR}=2.14)$, but was protective against depression for males $(\mathrm{OR}=0.31)$. In another study, Siegel et al., ${ }^{12}$ who controlled for demographic factors, found overweight is associated with symptoms of depression, a relationship that is partially explained by health status, but not by dietary restraint. Using multiple regression, poor health status, alcohol intake, hostility, and low levels of ethnic identity (connectedness with the AfricanAmerican community) were found to be independent predictors of depressed mood. In this study, dietary restraint reflected healthful eating, which was inversely related to depression. The overweight/ depression association was stronger among women with high levels of education and high levels of ethnic identity.

Traversy et al., ${ }^{9}$ concluded that despite the important limitations of current studies on this topic, it appears reasonable to state that alcohol intake may be a risk factor for obesity in some individuals, especially women. Shelton et al., ${ }^{13}$ who investigated the contribution of alcoholderived calories to the alcohol-obesity relation found that the mean alcohol calorie consumption was $27 \%$ of the recommended daily calorie intake in men and 19\% in women on the heaviest drinking day in the last week, with a positive association between alcohol calories and obesity. It was concluded that alcohol based calories may be a significant contributor to the rise in obesity. Although Constant et al., ${ }^{14}$ found a negative association between over eating due to distress and alcohol use in normal weight college aged women, Mason et al., ${ }^{15}$ found overweight and obesity were associated with more public identification as a lesbian, more depressive symptoms, increased heavy drinking, longer relationship length, and lower relationship consensus.

\section{Discussion}

Adult obesity, especially among women is a major health challenge with few solutions. ${ }^{7}$ One factor that has not been well explored is a possible link between alcohol, depression, and obesity. These aforementioned data, presented here, though limited, and not always consistently replicated [eg., $]^{14,16}$ appear sufficient to imply that there may be a link between alcoholism, or excess alcohol intake, and obesity in some women and possibly in men that would be valuable to explore, even if alcoholic intake alone is not a key obesity determinant in women. The link between alcohol, depression, and obesity among women, is also a possible determinant of obesity among women that is not well understood. In absence of definitive conclusions regarding women's high obesity rates, these linkages have emerged in recent years, as possible obesity correlates in women, and the fact no obesity prevalence changes have been observed, despite many other theories and follow-up intervention attempts among women. Perhaps women are more vulnerable to both a changing food and cultural environment, as well having a high predisposition to stress, alcoholism and related disorders, social, economic, and emotional factors. Food addiction and impulsivity is also predicted by alcohol use, ${ }^{17}$ but very few studies examined both these factors simultaneously. Since alcoholics, as compared with social drinkers, showed a lower body weight due essentially to a fat mass reduction, ${ }^{18}$ it seems other explanations for an alcohol-obesity linkage must be considered though, such as media glamorization of alcohol, more high level stressors incurred by high income and low income women in recent years, and social patterns of drinking behaviors that are more normative today.

In addition, as in other addictive behaviors, it is possible that the chronic use of alcohol, with or without certain foods, along with the possible resultant lack of engagement in physical activity tends to result in weight gain, rather than weight loss or maintenance. In women, the ensuing high level of body dissatisfaction that may follow, along with an emerging poor self-concept, and reduced emotional wellbeing, plus other health, social, and quality of life problems may foster depression, along with an inability to readily reverse this situation. ${ }^{19}$

Other explanations for an alcohol, obesity, depression link include, but are not limited to:

a. Sociological factors-that prevail in early childhood and beyond, such as trauma.

b. Biological factors, such as night work.

c. Sleep disturbances due to other factors

d. A neurobiological link between alcohol use and food consumption, consistent with theories characterizing excess food consumption as an addictive behavior. ${ }^{20}$

e. Alcohol may function as a depressant.

f. Preexisting psychiatric disorders.

g. Alcohol is associated with pleasurable sensations.

h. Ruminative coping leading to depression and binge drinking or eating as a coping mechanism.

i. Using eating and drinking as a reward mechanism.

j. Alcohol is socially acceptable and glorified in the media.

k. Psychological variables and eating style may predict alcohol consumption in obese individuals ${ }^{17}$

1. Alcohol may be deemed satisfying to the brain, thus making it highly sought after, as proposed by Mogul et al..$^{21}$

m. Alcohol use may alter cognitions, and ability to monitor/change behaviors readily.

n. Need for increased amounts alcohol to achieve intoxication or desired effect over time. Results of one or more of these factors are depicted in Figure 1.

\section{Obesity}
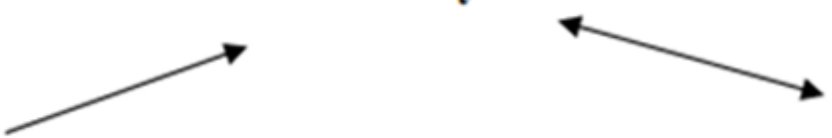

\section{Food/alcohol excess}

Figure I Hypothetical Associations between Obesity, Alcohol Intake, and Depression in women. 


\section{Implications}

Although more research is clearly needed, several solutions advocated for reducing the possible alcohol, depression, obesity linkage among women, via various approaches applied independently or collectively may be helpful to some in the efforts to curtail obesity rates among women, and possibly men. These include, but are not limited to:

Empowerment programs, cognitive reframing strategies, stress reduction/management programs, support groups, exercise, mindfulness meditation, avoiding alcohol as a coping mechanism, and counseling. Treating depression, obesity, and alcohol abuse simultaneously may also be helpful. Those who are most vulnerable should be screened and followed up accordingly.

\section{Conclusion}

There is clearly no abatement in the ongoing global obesity pandemic, wherein the prevalence of overweight and obesity among men and women varies, but overall, more women are obese than men. ${ }^{2}$ This state is not however, inevitable at all, but is more often than not linked to life styles and behaviors among other factors, including eating behaviors, and poor stress control. The significance of exploring the relationship depicted in this present overview is manifold, given the enormous challenges posed by the presence of obesity both individually and collectively, and the fact the obesity epidemic cannot be resolved readily by any vaccine or pill, or current recommendations to render changes in lifestyle and behavior such as exercise and eating behaviors, in most cases, without examining a broader array of sociocultural factors. Capturing this need very aptly in this author's view is the statement by Dubay et al., ${ }^{22}$ which states: There is simply no mistaking the reality that American women are currently dying much earlier than their counterparts in other advanced nations ... [including] women of reproductive and child rearing ages, a finding that has huge implications for children, families and communities... [and if obesity is one factor that is modifiable in this respect, as proposed by Cready, ${ }^{23}$ much more attention is warranted]. We thus urge researchers and clinicians to carefully examine all lifestyle factors, as well as cognitions in additional to the more traditional modifiable obesity determinants of their female clients, so that more can be said about what accounts for the increasing trend in obesity prevalence, especially among women, and what can be done proactively to limit the risk of obesity among women, in particular. After that, intervention needs can be better clarified, and, where possible lifestyles or other forms of intervention can be introduced to modify or obviate the rising obesity trends, ${ }^{24}$ and the interactions depicted in Figure 1. Since one of these trends may be the increase in recreational use of alcohol, another, the alcohol depression linkage, another the glorification of alcohol, or cognitive outcomes of alcohol use to reduce stress, more attention to this topic, especially a careful analysis of female clients during primary care visits, and salient follow up strategies are highly indicated. As well, marketing companies and restaurants should legally have to provide explicit salient information on the caloric implications of all alcohol based refreshments. Since obesity is a major contributor to some of the leading causes of premature death and excess morbidity in the US and elsewhere, including heart disease, stroke, diabetes and some types of cancer and osteoarthritis, non-profit organizations that serve these clients, should make the dangers of excess alcohol consumption as regards caloric intake much more visible. Women's groups too can advocate for marketing messages that are evenly balanced in depicting the benefits and pitfalls of excess alcohol consumption on weight gain. The importance of treating depression, and food addictions, including alcohol addiction should also receive more attention in the media and clinical literature. Groups where binge drinking is a social 'norm' should also be apprised of the dangers to women, in particular of alcohol excess, given that that alcohol calories count more in moderate nondaily consumers than in daily (heavy) consumers, especially in those individuals who consume high-fat diet and in overweight and obese subjects..$^{25}$

\section{Acknowledgments}

None.

\section{Conflicts of interest}

The author declares there are no conflicts of interest.

\section{References}

1. What are overweight and obesity? National Heart Blood and Lung Institute. 2015.

2. Kanter R, Caballero B. Global gender disparities in obesity: a review. $A d v$ Nutr. 2012;3(4):491-498.

3. Blumenthal S, Levin S. Global Obesity: A Growing Epidemic. 2015.

4. Obesity and Overweight (WHO). 2015.

5. Overweight and Obesity. Minority Women's Health.

6. Grucza RA, Krueger RF, Racette SB, et al. The emerging link between alcoholism risk and obesity in the United States. Arch Gen Psychiatry. 2010;67(12):1301-1308.

7. Charo L, Lacoursiere DY. Introduction: obesity and lifestyle issues in women. Clin Obstet Gynecol. 2014;57(3) :433-445.

8. CDC/NCHS. National Health and Nutrition Examination Survey, 20052010.

9. Traversy G, Chaput JP. Alcohol consumption and obesity: an update. Curr Obes Rep. 2015;4(1):122-130.

10. Maksimović MŽ, Gudelj Rakić JM, Vlajinac HD, et al. Relationship between health behaviour and body mass index in the Serbian adult population: data from National Health Survey 2013. Int J Public Health. 2016;61(1):57-68.

11. McCarty CA, Kosterman R, Mason WA, et al. Longitudinal associations among depression, obesity and alcohol use disorders in young adulthood. Gen Hosp Psychiatry. 2009;31(5):442-450.

12. Siegel JM, Yancey AK, McCarthy WJ. Overweight and depressive symptoms among African-American women. Prev Med. 2000;31(3):232240.

13. Shelton NJ, Knott CS. Association between alcohol calorie intake and overweight and obesity in English adults. Am J Public Health. 2014;104(4):629-631.

14. Constant A, Gautier Y, Coquery N, et al. Emotional overeating is common and negatively associated with alcohol use in normal-weight female university students. Appetite. 2018;16;129:186-191.

15. Mason TB, Lewis RJ. Minority stress, depression, relationship quality, and alcohol use: associations with overweight and obesity among partnered young adult lesbians. LGBT Health. 2015;2(4):333-340.

16. Jia H, Zack MM, Gottesman II, et al. Associations of smoking, physical inactivity, heavy drinking, and obesity with quality-adjusted life expectancy among us adults with depression. Value Health. 2018;21(3):364-371. 
17. Meule A, Heckel D, Jurowich CF. Correlates of food addiction in obese individuals seeking bariatric surgery. Clin Obes. 2014;4(4):228-236.

18. Addolorato G, Capristo E, Greco AV, et al. Influence of chronic alcohol abuse on body weight and energy metabolism: is excess ethanol consumption a risk factor for obesity or malnutrition? J Intern Med. 1998;244(5):387-395.

19. Shriner R, Gold M. Food addiction: an evolving nonlinear science. Nutrients. 2014;6(11):5370-5391.

20. Gearhardt AN, Corbin WR. Body mass index and alcohol consumption: family history of alcoholism as a moderator. Psychol Addict Behav. 2009;23(2):216-225.
21. Mogul A, Irby MB, Skelton JA. A systematic review of pediatric obesity and family communication through the lens of addiction literature. Child Obes. 2014;10(3):197-206.

22. Dubay L, Waxman E, Martin S. To understand climbing death rates among Whites, look to women of childbearing age. Health Affairs Blog. 2015.

23. Cready G. Alcoholism and Obesity.

24. Baik I. Forecasting obesity prevalence in Korean adults for the years 2020 and 2030 by the analysis of contributing factors. Nutr Res Pract. 2018;12(3):251-257.

25. Suter PM. Is alcohol consumption a risk factor for weight gain and obesity? Crit Rev Clin Lab Sci. 2005;42(3):197-227. 